\title{
Separation of cyclic and starlike hierarchical dominance in evolutionary matrix games
}

\author{
György Szabó, ${ }^{1}$ Kinga S. Bodó, ${ }^{2}$ and Keivan Aghababaei Samani ${ }^{3}$ \\ ${ }^{1}$ Institute of Technical Physics and Materials Science, Centre for Energy Research, Hungarian Academy of Sciences, \\ P.O. Box 49, H-1525 Budapest, Hungary \\ ${ }^{2}$ Budapest University of Technology and Economics, Budafoki út 8, H-1111 Budapest, Hungary \\ ${ }^{3}$ Department of Physics, Isfahan University of Technology, Isfahan 84156-83111, Iran
}

(Received 10 November 2016; published 23 January 2017)

\begin{abstract}
We study antisymmetric components of matrices characterizing pair interactions in multistrategy evolutionary games. Based on the dyadic decomposition of matrices we distinguish cyclic and starlike hierarchical dominance in the appropriate components. In the symmetric matrix games the strengths of these elementary components are determined. The general features and intrinsic symmetries of these interactions are represented by directed graphs. It is found that the variation of a single matrix component modifies simultaneously the strengths of two starlike hierarchical basis games and many other independent rock-paper-scissors type cyclic basis games. The application of the related concepts is illustrated by discussing the three-strategy voluntary prisoner's dilemma.
\end{abstract}

DOI: 10.1103/PhysRevE.95.012320

\section{INTRODUCTION}

In evolutionary games payoff matrices characterize the pair interactions in the multiagent models describing biological and social systems [1-7]. The evolutionary processes and stationary states of these systems are strongly influenced by these interactions as well as by the applied dynamical rule and connectivity structure. For symmetric games the players are equivalent and the interactions are defined by a single payoff matrix $\mathbf{A}$ where $A_{i j}$ denotes the payoff of the first player if she chooses her $i$ th strategy while her coplayer selects the $j$ th one ( $1 \leqslant i, j \leqslant n$ for $n$ strategies). The classification of these matrix interactions into different types $[8,9]$ efficiently helps the systematic investigation of the possible behaviors that is complicated by the large number of payoff parameters.

The decomposition of matrices into the linear combination of orthogonal matrices has thrown light on the inherent symmetries and indicated possibilities for different classifications. For example, if the elementary orthogonal set of matrices are built up from the dyadic products of two sets of orthogonal basis vectors then we can easily distinguish the games with self- and cross-dependent payoffs [10] which represent the absence of direct pair interactions between the players. In those cases one of the basis vectors of the dyadic products is a vector with all components being 1 . The corresponding set of matrices is built up from rows (or columns) with uniform values; that is, $A_{i j}=\varepsilon_{i}$ for the self-dependent components (or $A_{i j}=\delta_{j}$ for the cross-dependent games). For example, the payoff matrix of the donation game [11] (or simplified prisoner's dilemma game [6]) can be composed of a self- and a cross-dependent component. The additional orthogonal terms of the payoff matrix can be separated into symmetric and antisymmetric components [10]. It is found that the symmetric components can be considered as linear combinations of coordination type interactions between any possible strategy pairs as described in a recent paper [12]. We have to emphasize that all these elementary coordination games are equivalent and can be mapped onto each other by relabeling the strategy indices.

It is worth noting that the symmetric games with symmetric payoff matrices $A_{i j}=A_{j i}$ are potential games and these systems exhibit thermodynamical behavior if the logit rule controls the evolution of the strategy distribution [13-18]. In that case the potential matrix is equivalent to the payoff matrix. The latter quantity summarizes the payoff variation of the active player when moving in the strategy space via consecutive unilateral strategy changes. In fact, the name of potential refers to the negative potential energy in physical systems (like the Ising model).

The payoff matrices of the game theory, however, may be asymmetric. At the same time all these matrices can be decomposed into the sum of symmetric and antisymmetric parts as $\mathbf{A}=\mathbf{A}^{(\mathrm{s})}+\mathbf{A}^{(\mathrm{as})}$, where $\mathbf{A}^{(\mathrm{s})}=\left(\mathbf{A}+\mathbf{A}^{T}\right) / 2, \mathbf{A}^{(\mathrm{as})}=$ $\left(\mathbf{A}-\mathbf{A}^{T}\right) / 2$, and the index $T$ refers to transpose. Now we study the internal structure of the antisymmetric part that can be built up as the sum of two types of games, namely, $\mathbf{A}^{(\mathrm{as})}=\mathbf{A}^{(\mathrm{c})}+\mathbf{A}^{(\mathrm{h})}$, where the upper labels denote cyclic and hierarchical dominance. These games are symmetric zero sum games where both players receive nothing if they choose the same strategy $\left(A_{i i}^{(\text {as })}=0\right)$ while when selecting different strategies one gains and the other loses an equivalent amount $\left(A_{i j}^{\text {(as) }}=-A_{j i}^{\text {(as) }}\right)$. It has previously been shown that there are $(n-1)(n-2) / 2$ independent cyclic components that prevent the existence of potential $[7,9]$. Now we show that the set of rock-paper-scissors type elementary games with three suitable strategies (namely, with $1, i$, and $j$ where strategy 1 dominates strategy $i$ dominating $j$ dominating 1 with equal strength and $1<i<j \leqslant n)$ spans the parameters space of $\mathbf{A}^{(\mathrm{c})}$. In addition, the antisymmetric matrix interactions involve $(n-1)$ starlike hierarchical components that are derived from the selfand cross-dependent games. The latter type of interactions is responsible for the occurrence of social dilemmas [7,11].

In the next sections we describe the possible and independent components of the antisymmetric matrix interactions and we show how the strengths of these independent components can be evaluated. The general features and symmetries of the antisymmetric elementary matrices are illustrated by an appropriate set of directed graphs. When considering the linear combinations of these elementary components or graphs we get a picture about the nature of hierarchical dominance and also about how the large number of cyclic components are entangled. 


\section{ANTISYMMETRIC COMPONENTS DERIVED FROM DYADIC PRODUCTS}

The dyadic products of an orthogonal set of $n$-dimensional basis vectors $[\mathbf{v}(1), \mathbf{v}(2), \ldots, \mathbf{v}(n)$ with a scalar product $\mathbf{v}(p)$. $\mathbf{v}(q)=0$ if $p \neq q$ ] serve as an orthogonal set of basis matrices [7,9]. Consequently, any matrix $\mathbf{A}$ can be expressed as

$$
\mathbf{A}=\sum_{p, q=1}^{n} \frac{\alpha(p, q)}{c(p, q)} \mathbf{g}(p, q),
$$

where the dyadic products

$$
\mathbf{g}(p, q)=\mathbf{v}(p) \otimes \mathbf{v}(q)
$$

represent the orthogonal set of basis matrices with elements $g_{i j}(p, q)=v_{i}(p) v_{j}(q)$ for $p, q, i, j=1, \ldots, n$. If these matrices are considered as $n^{2}$-dimensional vectors then the matrices $\mathbf{A}$ and $\mathbf{A}^{\prime}$ are orthogonal to each other when their scalar product is zero; that is, $\mathbf{A} \cdot \mathbf{A}^{\prime}=\sum_{i, j} A_{i j} A_{i j}^{\prime}=0$. The coefficients in Eq. (1) can be expressed by these scalar products as

$$
\alpha(p, q)=\mathbf{A} \cdot \mathbf{g}(p, q)
$$

and

$$
c(p, q)=\mathbf{g}(p, q) \cdot \mathbf{g}(p, q) .
$$

The decomposition (1) becomes meaningful if the first vector is chosen to be an all-ones vector (i.e., $v_{i}(1)=1 \forall i$ ). In these cases the self-dependent part of $\mathbf{A}$ is expressed by the sum of $n$ terms as

$$
\mathbf{A}^{(\mathrm{se})}=\sum_{p=1}^{n} \frac{\alpha(p, 1)}{c(p, 1)} \mathbf{g}(p, 1)=\left(\begin{array}{cccc}
\varepsilon_{1} & \varepsilon_{1} & \cdots & \varepsilon_{1} \\
\varepsilon_{2} & \varepsilon_{2} & \cdots & \varepsilon_{2} \\
\vdots & \vdots & \ddots & \vdots \\
\varepsilon_{n} & \varepsilon_{n} & \cdots & \varepsilon_{n}
\end{array}\right)
$$

and it is composed of rows with uniform payoffs as indicated. The cross-dependent components $\mathbf{A}^{(\mathrm{cr})}$ obey a similar expression, namely,

$$
\mathbf{A}^{(\mathrm{cr})}=\sum_{q=1}^{n} \frac{\alpha(1, q)}{c(1, q)} \mathbf{g}(1, q)=\left(\begin{array}{cccc}
\delta_{1} & \delta_{2} & \cdots & \delta_{n} \\
\delta_{1} & \delta_{2} & \cdots & \delta_{n} \\
\vdots & \vdots & \ddots & \vdots \\
\delta_{1} & \delta_{2} & \cdots & \delta_{n}
\end{array}\right),
$$

where the payoffs are the same in each column.

Notice that the all-ones matrix belongs to both the self- and cross-dependent components. The corresponding term $\mathbf{A}^{(\mathrm{av})}$ plays a distinguished or irrelevant role and can be expressed by the average value of payoffs. Quantitatively,

$$
\mathbf{A}^{(\mathrm{av})}=a^{(\mathrm{av})} \mathbf{g}(1,1), \quad \text { where } a^{(\mathrm{av})}=\frac{1}{n^{2}} \sum_{i, j} A_{i j} .
$$

Consequently, the dimension of the unified self- and crossdependent components is $2 n-1$. In the above formulas the matrix elements are average values in the suitable row or column; that is,

$$
\varepsilon_{i}=\frac{1}{n} \sum_{j} A_{i j} \quad \text { and } \quad \delta_{i}=\frac{1}{n} \sum_{j} A_{j i},
$$

which satisfy additional criteria:

$$
a^{(\mathrm{av})}=\frac{1}{n} \sum_{i} \varepsilon_{i}=\frac{1}{n} \sum_{i} \delta_{i} .
$$

First we discuss the hierarchical component $\mathbf{A}^{(\mathrm{h})}$ derived from the self- and cross-dependent games as

$$
\mathbf{A}^{(\mathrm{h})}=\frac{1}{2}\left(\mathbf{A}^{(\mathrm{se})}+\mathbf{A}^{(\mathrm{cr})}-\mathbf{A}^{(\mathrm{se}) \mathrm{T}}-\mathbf{A}^{(\mathrm{cr}) \mathrm{T}}\right) .
$$

Evidently the symmetric $\mathbf{A}^{(\mathrm{av})}$ does not give contribution to $\mathbf{A}^{(\mathrm{h})}$. The proper structures of $\mathbf{A}^{\text {(se) }}$ and $\mathbf{A}^{\text {(cr) }}$ imply the selection of a more suitable set of basis games that can also be expressed by dyadic products as

$$
\mathbf{H}(p)=\mathbf{u}(p) \otimes \mathbf{v}(1)-\mathbf{v}(1) \otimes \mathbf{u}(p),
$$

where $\mathbf{u}(p)(p=1, \ldots, n)$ are the traditional Cartesian unit vectors [with components $u_{i}(p)=\delta_{i p}$ defined by the Kronecker delta]. For this elementary game the player wins 1 from her coplayer if she chooses the $p$ th strategy and the coplayer chooses any other strategies while both players receive zero otherwise. In matrix notation

$$
\mathbf{H}(1)=\left(\begin{array}{ccccc}
0 & 1 & 1 & \ldots & 1 \\
-1 & 0 & 0 & \ldots & 0 \\
-1 & 0 & 0 & \ldots & 0 \\
\vdots & \vdots & \vdots & \ddots & \vdots \\
-1 & 0 & 0 & \ldots & 0
\end{array}\right)
$$

and all the other $\mathbf{H}(p)$ matrices can be obtained by exchanging the first and $p$ th rows and columns of $\mathbf{H}(1)$ simultaneously. The pure Nash equilibrium of this potential game is to choose the $p$ th strategy for both players when each gets zero payoff. The self- and cross-dependent games are potential games [7,10] for which one can derive a potential matrix $\mathbf{V}$ summarizing the individual interest for consecutive unilateral strategy changes. One can easily check that the potential matrix of $\mathbf{H}(p)$ can be expressed by Kronecker deltas as $V_{i j}^{(\mathrm{h})}(p)=\delta_{i p} \delta_{j p}$.

The matrices $\mathbf{H}(p)$ are not orthogonal to each other because $\mathbf{H}(p) \cdot \mathbf{H}\left(p^{\prime}\right)=-2$ if $p \neq p^{\prime}$, whereas all these $\mathbf{H}(p)$ matrices are orthogonal to the symmetric parts of $\mathbf{A}$ and to the cyclic components discussed later. The scalar product of $\mathbf{A}$ and $\mathbf{H}(p)$ depends only on the payoff parameters $\varepsilon_{p}-\delta_{p}$ because

$$
\begin{aligned}
e_{p} & =\frac{1}{n} \mathbf{H}(p) \cdot \mathbf{A}=\frac{1}{n} \mathbf{H}(p) \cdot \mathbf{A}^{(\mathrm{h})} \\
& =\frac{1}{n} \sum_{i}\left(\varepsilon_{p}-\varepsilon_{i}+\delta_{i}-\delta_{p}\right) \\
& =\varepsilon_{p}-\delta_{p}
\end{aligned}
$$

and $e_{p}$ quantifies the asymmetry and it is equivalent to the average payoff the player wins with using the $p$ th strategy from the other player selecting her strategy at random. Evidently,

$$
\sum_{p} e_{p}=0
$$

The positive or negative $e_{p}$ refers to a greedy or losing strategy and this quantity can rank the strategies from the viewpoint of greediness. 
The whole set of the $\mathbf{H}(p)$ matrices are not independent of each other because

$$
\sum_{p=1}^{n} \mathbf{H}(p)=0 .
$$

On the other hand, any $(n-1)$ terms of these $\mathrm{H}(p)$ matrices span the space of hierarchical games $\mathbf{A}^{(\mathrm{h})}$. The reader can easily check that the hierarchical component is given as a linear combination of all $\mathbf{H}(p)$ matrices; that is,

$$
\mathbf{A}^{(\mathrm{h})}=\frac{1}{2} \sum_{p=1}^{n} e_{p} \mathbf{H}(p) .
$$

In this expression Eq. (14) ensures that the number of independent parameters remains $(n-1)$. Using Eq. (15), however, one can derive other linear combinations for $\mathbf{A}^{(\mathrm{h})}$. For example,

$$
\mathbf{A}^{(\mathrm{h})}=\frac{1}{2} \sum_{p=1}^{n}\left(e_{p}-\mu\right) \mathbf{H}(p)
$$

may also be a suitable expression. In that case, the sum of the prefactors differs from zero if $\mu \neq 0$. On the other hand, if $\mu=e_{j}$ then the contribution of $\mathbf{H}(j)$ is replaced by other components in agreement with Eq. (15).

The above simple formulas are related to the fact that the $\mathbf{H}(p)$ basis games are described by sparse matrices. Now we exploit the same feature in the analysis of the cyclic components.

In the notation of dyadic decomposition $[7,10]$ the coordination $\left(\mathbf{A}^{(\mathrm{co})}\right)$ and cyclic $\left(\mathbf{A}^{(\mathrm{cy})}\right)$ components of symmetric matrix games are defined as

$$
\mathbf{A}^{(\mathrm{co})}=\sum_{\substack{p, q \\ 1<p<q}}^{n} \beta(p, q)[\mathbf{g}(p, q)+\mathbf{g}(q, p)]
$$

and

$$
\mathbf{A}^{(\mathrm{cy})}=\sum_{\substack{p, q \\ 1<p<q}}^{n} \gamma(p, q)[\mathbf{g}(p, q)-\mathbf{g}(q, p)],
$$

where

$$
\begin{aligned}
& \beta(p, q)=\frac{1}{2 c(p, q)}[\alpha(p, q)+\alpha(q, p)], \\
& \gamma(p, q)=\frac{1}{2 c(p, q)}[\alpha(p, q)-\alpha(q, p)] .
\end{aligned}
$$

The matrices $\mathbf{A}^{(\mathrm{co})}$ and $\mathbf{A}^{(\mathrm{cy})}$ are orthogonal to each other as well as to the self- and cross-dependent components defined by Eqs. (5) and (6). Previous analysis has justified that for $n=3$ we have only one cyclic component that is equivalent to the rock-paper-scissors game [9], while for $n=4, \mathbf{A}^{\text {(cy) }}$ can be built up as a linear combination of three rock-paper-scissors type interactions [10]. For the latter case one can find a fourth rock-paper-scissors component and also two additional four-strategy cyclic games. The latter feature is related to the inherent symmetry resembling the three-dimensional cubic lattices where we have four equivalent (space diagonal) unit vectors.
Now we show algebraically that for $n$ strategies we can introduce $(n-1)(n-2) / 2$ rock-paper-scissors type components that span the subspace of cyclic components. The corresponding matrices are denoted by $\mathbf{C}(1, k, l)$ and describe rock-paper-scissors type cyclic dominance among the denoted three strategies; that is,

$$
C_{i j}(1, k, l)=\left\{\begin{aligned}
1 & \text { if } i=1 \text { and } j=k \\
1 & \text { if } i=k \text { and } j=l \\
1 & \text { if } i=l \text { and } j=1 \\
-1 & \text { if } j=1 \text { and } i=k \\
-1 & \text { if } j=k \text { and } i=l \\
-1 & \text { if } j=l \text { and } i=1 \\
0 & \text { otherwise, }
\end{aligned}\right.
$$

where $1<k<l \leqslant n$. Although some of the $\mathbf{C}(1, k, l)$ matrices are not orthogonal to each other [e.g., $\left.\mathbf{C}(1, k, l) \cdot \mathbf{C}\left(1, k, l^{\prime}\right) 2\right]$, their linear combinations obey the following simple form:

$$
\sum_{\substack{k, l \\
1<k<l}}^{n} v_{k l} \mathbf{C}(1, k, l)=\left(\begin{array}{ccccc}
0 & \varrho_{2} & \varrho_{3} & \cdots & \varrho_{n} \\
-\varrho_{2} & 0 & v_{23} & \cdots & v_{2 n} \\
-\varrho_{3} & -v_{23} & 0 & \cdots & v_{3 n} \\
\vdots & \vdots & \vdots & \ddots & \vdots \\
-\varrho_{n} & -v_{2 n} & -v_{3 n} & \cdots & 0
\end{array}\right),
$$

where

$$
\varrho_{k}=\sum_{l=2}^{k} v_{k l}-\sum_{l=k+1}^{n} v_{k l} .
$$

Notice that in the above linear combination the basis matrix $\mathbf{C}(1, k, l)$ gives a contribution $v_{k l}$ exclusively to $A_{k l}^{(\mathrm{cy})}$ if $1<$ $k<l \leqslant n$, and the values of $\varrho_{k}$ (in the first row and column of $\left.A^{(\mathrm{cy})}\right)$ ensure that the sum of the matrix elements is zero in each row and column. That means that $A_{k l}^{\text {(cy) }}=v_{k l}(1<k<l \leqslant n)$, and this expression gives us a simple method for the identification of the coefficients $v_{k l}$ in the knowledge of the cyclic component $A^{(\mathrm{cy})}$.

\section{GRAPH REPRESENTATION}

Directed graphs [19] can be used to illustrate the general features of the symmetric zero-sum games because their adjacency matrices define a suitable set of basis matrices. In the simplest case the graphs $\mathcal{G}(i, j)$ with $n$ nodes and a single directed edge from the $i$ th node to the $j$ th one (see Fig. 1) represent a natural set of basis matrices or games where the matrix elements of the adjacency matrices are zero except when $G_{i j}(i, j)=-G_{j i}(i, j)=1$. Henceforth, we use calligraphic letters (e.g., $\mathcal{G}$ ) to indicate a directed graph defined by an antisymmetric adjacency matrix $\mathbf{G}$.

In this notation the antisymmetric part of the payoff matrix is given as $\mathbf{A}^{\text {(as) }}=\sum_{i, j} A_{i j}^{\text {(as) }} \mathbf{G}(i, j)$. In fact, the matrix $\mathbf{A}^{\text {(as) }}$ can be considered as the adjacency matrix of a weighted directed graph where $A_{i j}$ quantifies the weight of the given directed edge. If all the directions of existing edges are reversed in a directed graph $\mathcal{G}$ then the sign of the adjacency matrix is reversed, e.g., $\mathbf{G}(i, j) \rightarrow \mathbf{G}(j, i)=-\mathbf{G}(i, j)$.

The adjacency matrices $\mathbf{G}(i, j)$ (with $1 \leqslant i<j \leqslant n$ ) define a complete orthogonal basis for $\mathbf{A}^{\text {(as) }}$. In the previous section 


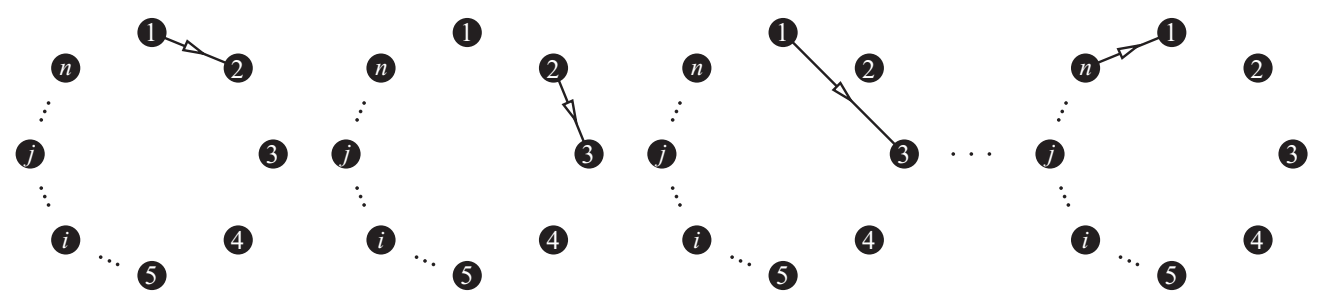

FIG. 1. Set of directed graphs $\mathcal{G}(i, j)$ with a single directed edge from the node $i$ to node $j$.

we have shown that instead of this natural basis we can use another complete set of basis matrices composed of $\mathbf{H}(p)$ $(1<p \leqslant n)$ and $\mathbf{C}(1, k, l)(1<k<l \leqslant n)$ that can also be represented by directed graphs, as illustrated in Figs. 2 and 3.

The hierarchical basis matrix $\mathbf{H}(p)$ is identical to the adjacency matrix of a directed starlike graph $\mathcal{H}(p)$ which has directed edges from the $p$ th node to all the others.

These graphs (as well as their adjacency matrices) can be considered as a suitable sum of the graphs $\mathcal{G}(i, j)$. To be more quantitative, now the sum of two directed graphs $\left(\mathcal{G}\right.$ and $\mathcal{G}^{\prime}$ with the same labeled nodes) is defined by combining the two sets of edges. In this approach the opposite edges annihilate each other and parallel edges are doubled. The reader can check that the sum of the starlike hierarchical graphs, plotted in Fig. 2, results in an empty graph (with $n$ nodes without edges).

The application of this summation rule is visualized in Fig. 4, which shows how any directed loop (or the sum of directed loops) can be built up from a suitable combination of those directed three-edge loops including node 1 (plotted in Fig. 3).

In words, directed three-edge loops are obtained by connecting both ends of each edge of a directed loop to node 1 and the ingoing and outgoing edges of node 1 will annihilate each other in the corresponding sum of the resulted directed triangles.

We emphasize that the numbers of three- and $n$-edge directed loops are significantly larger than the number of the independent basis cyclic graphs $\mathcal{C}(1, i, j)$. The cyclic graphs are entangled via their common edges. Thus if the system contains only a single basis cyclic component [e.g., $\mathbf{C}(1,2,3)]$ then there appear many other cyclic components [e.g., $\mathbf{C}(1,2, k), \mathbf{C}(1, k, 3)$, and $\mathbf{C}(k, 2,3)$, with $k>3$ ] that influence the evolutionary processes.

For all the possible combinations of the directed threeedge loops $\mathcal{C}(1, i, j)$ the numbers of ingoing and outgoing edges are equivalent for each node. This feature ensures the orthogonality of the corresponding adjacency matrices to both $\mathbf{A}^{(\mathrm{se})}$ and $\mathbf{A}^{\text {(cr) }}$ from which the starlike hierarchical basis graphs (see Fig. 2) are derived. Thus we can speak of two orthogonal graphs if the scalar product of their adjacency matrices is zero. In this sense the single-edge graphs plotted in Fig. 1 are orthogonal to each other. Similarly, each starlike graph (see Fig. 2) is orthogonal to any other cyclic graphs plotted in Figs. 3 and 4.

The concept of the complete independent set of basis matrices can be adapted to introduce the complete and independent set of basis graphs from which any directed graph can be constructed by linear combinations. Indeed, the single-edge directed graphs (see Fig. 1) serve as a standard choice.

In Fig. 5 we show that any single edge graph $\mathcal{G}(i, j)$ can be considered as a sum of $\mathcal{H}(p)$ and $\mathcal{C}(i, j, k)$ graphs.

More accurately, Fig. 5 illustrates the graph representation of the relation

$$
n \mathbf{G}(i, j)=\mathbf{H}(i)-\mathbf{H}(j)+\sum_{\substack{k \\ k \neq i, j}} \mathbf{C}(i, j, k)
$$

that becomes correct when allowing multiple or weighted edges in the set of directed graphs. This formula obeys the following form:

$$
\begin{aligned}
n \mathbf{G}(i, j)= & \mathbf{H}(i)-\mathbf{H}(j)+(n-2) \mathbf{C}(1, i, j) \\
& +\sum_{\substack{k \\
k \neq 1, j, k}}[\mathbf{C}(1, k, i)+\mathbf{C}(1, k, j)]
\end{aligned}
$$

if all the three-edge cyclic graphs $\mathcal{C}(i, j, k)$ are built up only from those ones containing node 1 (see Fig. 4$)$, that is, when substituting the expression $\mathbf{C}(i, j, k)=\mathbf{C}(1, i, j)+\mathbf{C}(1, k, i)+$ $\mathbf{C}(1, j, k)$ into Eq. (23). Consequently, all the directed graphs can also be composed of the basis graphs $\mathcal{H}(p)$ and $\mathcal{C}(1, i, j)$.

In game theory the main message of Eq. (23) and Fig. 5 is that the variation of $\mathbf{A}_{i j}^{(\text {as })}$ is shared equally among two hierarchical and $(n-2)$ cyclic components including the strategies $i$ and $j$.
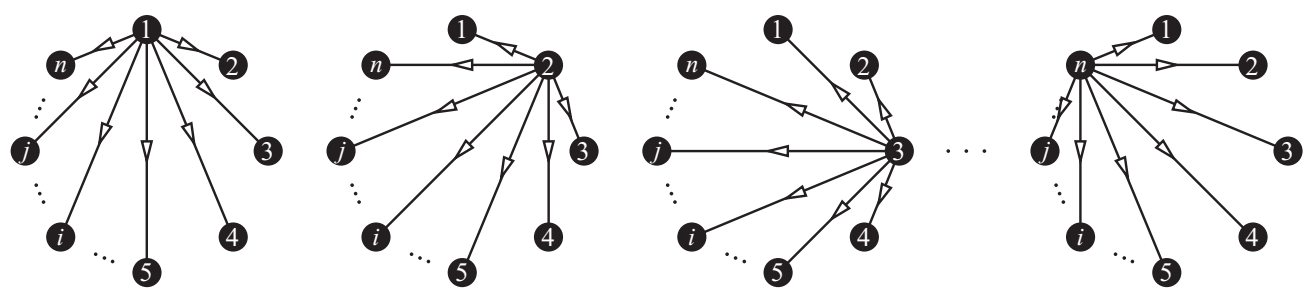

FIG. 2. Directed graphs representing starlike hierarchical dominance. Except for one of them these graphs define $(n-1)$ independent (nonorthogonal) basis games $\mathcal{H}(p)$. 


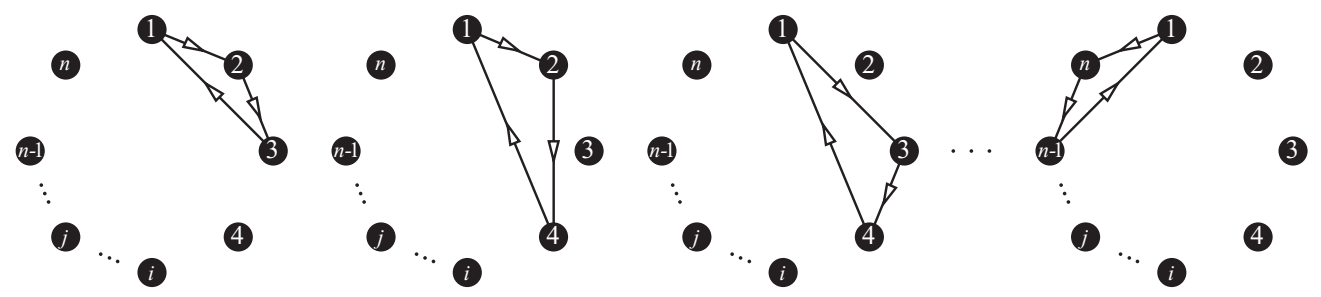

FIG. 3. Directed three-edge loops including node 1 define $(n-1)(n-2) / 2$ independent cyclic basis games $\mathbf{C}(1, i, j)$ as described in the text.

\section{EXAMPLE: VOLUNTARY PRISONER'S DILEMMA}

Now we demonstrate the evaluation of the strengths of the hierarchical and cyclic dominance by considering a wellinvestigated symmetric three-strategy game. The example is the voluntary prisoner's dilemma which is already studied for well-mixed populations and also on lattices and different networks [20-22]. In these games the players have three strategies: $D$ (defection), $C$ (cooperation), and $L$ (loner). Following the traditional notation of social dilemmas [3,11], the payoff matrix is defined by three parameters [22] as

$$
\mathbf{A}=\left(\begin{array}{lll}
0 & T & \sigma \\
S & 1 & \sigma \\
\sigma & \sigma & \sigma
\end{array}\right)
$$

If the players are constrained to use only the first $(D)$ and second $(C)$ strategies then the game is equivalent to the prisoner's dilemma. In the latter case both rational players are enforced to choose $D$ providing zero income (as it is the single pure Nash equilibrium), whereas for mutual cooperation both players would receive a higher income considered as a payoff unit. The "tragedy of the commons" [23,24] is caused by the high value of temptation $(T>1)$ to choose defection against the cooperative coplayer who receives a low sucker's payoff $(S<0)$. A large portion of papers in the literature of evolutionary game theory study methods developed to avoid this social dilemma. One of the suggested methods is related to the voluntary participation $[20,21]$ in the game via the introduction of the third strategy $L$. Accordingly, if one of the players chooses $L$ then both receive $\sigma$ with $0<\sigma<1$ [22].

The symmetric part of this payoff matrix predicts the $C C$ strategy pair to be the preferred Nash equilibrium if $(T+S) / 2<1$ [7]. In the opposite case $[(T+S) / 2<1]$ the game has two equivalent Nash equilibria $(D C$ and $C D$ ) characteristic of the anticoordination games. It is worth noting that the strategy pair $L L$ is a weak Nash equilibrium with a low value of potential; therefore, it plays an irrelevant role in the multiagent evolutionary game if the evolution is controlled by logit rule for a low noise level.

The curiosity of these three-strategy systems is that both the social dilemma and the presence of a cyclic component are caused by a single pair of nonvanishing matrix elements of $\mathbf{A}^{(\text {as) }}$ that is given as

$$
\mathbf{A}^{(\mathrm{as})}=\frac{T-S}{2}\left(\begin{array}{rrr}
0 & 1 & 0 \\
-1 & 0 & 0 \\
0 & 0 & 0
\end{array}\right) .
$$

Figure 5 indicates that

$$
\mathbf{A}^{(\text {as })}=\frac{T-S}{6}(\mathbf{H}(1)-\mathbf{H}(2)+\mathbf{C}(1,2,3)) .
$$

The hierarchical components $[\mathbf{H}(1)-\mathbf{H}(2)]$ enforce the players to choose $D$. The corresponding greediness parameters

$$
e_{1}=-e_{2}=\frac{T-S}{3} \text { and } e_{3}=0
$$

indicate that the first strategy represents the greedy behavior, the second strategy plays the role of sucker, whereas the third one is neutral.

In this model the introduction of the third (loner) strategy is accompanied by the relevant presence of a rock-paper-scissors component that prevents the existence of potential as well as the thermodynamic behavior of this evolutionary game for the application of a logit rule. For imitation type dynamical rules $[3,4]$ the multiagent voluntary prisoner's dilemma on a square lattice exhibited a self-organizing pattern with rotating spiral arms [22,25,26]. In well-mixed populations [21,27] and also on some small-world networks [28-30] this interaction yielded global oscillations in the strategy frequencies. The "tragedy of the commons" is avoided in all these systems because the cyclic component supports the survival of all three strategies. Similar phenomena were reported in many models developed to explain the maintenance of biodiversity [4,31-36].

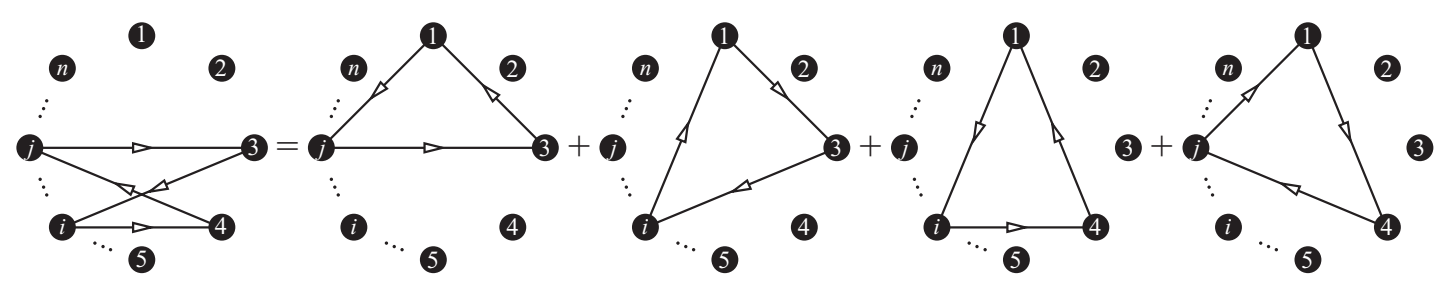

FIG. 4. Any directed loop can be considered as a sum of directed triangles involving node 1. In the sum of directed graphs coinciding two edges with opposite directions annihilate each other. 


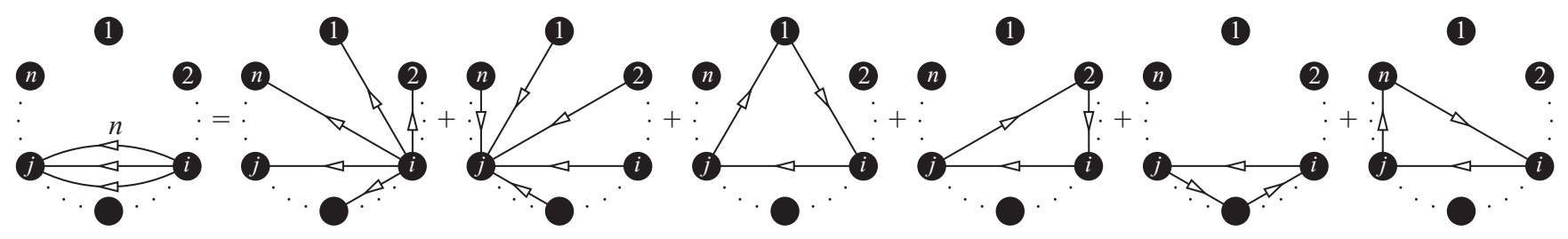

FIG. 5. The sum of two starlike directed graphs $[\mathcal{H}(i)-\mathcal{H}(j)]$ and $(n-2)$ directed triangles $[\mathcal{C}(i, j, k)$ with $k \neq i, j$ and $i<j]$. Notice that all these elementary graphs possess a directed edge from node $i$ to $j$.

In most of these systems the effect of cyclic dominance is affected weakly by the additional terms of interaction. Now we recall only a paradoxical effect described by Tainaka [37]. The increase of $T$ is beneficial for the first strategy; therefore, naively one can expect an increase in the defector population, too. Instead of it, both the numerical simulations on different networks and the analytical calculations predict an increase in the frequency of loners [22,26], in agreement with the explanation given in [37].

\section{SUMMARY}

The concept of the decomposition of the $n$-strategy symmetric matrix games into the sum of elementary basis games has opened ways for the classification of interactions determining the noise-dependent stationary states in multiagent evolutionary games. Mathematically this decomposition is equivalent to a suitable rotation of the coordinate system in the $n^{2}$-dimensional parameter space when the new basis matrices reflect well the inherent symmetries and features of interactions. Using this tool we can improve our picture of the effect of interactions on the final stationary state in complex systems [38] (including biology, economics, sociology, psychology, etc.) where these types of interactions are entangled.

Now our analyses are focused on the investigation of the antisymmetric parts of the payoff matrices quantifying the $n$-strategy zero sum games. Two fundamentally different types of antisymmetric basis matrices are distinguished. The hierarchical components are derived from the self- and crossdependent games defined by matrices with uniform values in rows and columns. This subset of games is spanned by $(n-1)$ hierarchical basis games that are equivalent to the adjacency matrices of starlike directed graphs.

The set of independent cyclic components is defined by rock-paper-scissors type games for three of $n$ strategies selected in a way that one of the distinguished strategies is included in all the $(n-1)(n-2) / 2$ independent cyclic components. The matrices of these elementary games are identical to adjacency matrices of directed graphs with $n$ nodes and a directed three-edge loop.

The intimate relationship between the antisymmetric matrices and directed graphs is used to demonstrate the inherent structure and symmetries hidden in interactions described by antisymmetric matrices. The graph representation has illustrated clearly the entanglement of loops and also how the large number of possible directed loops can be decomposed into independent elementary three-edge loops. Additionally, the graph representation has shown that the variation of a matrix element is accompanied by a change in two hierarchical and many other cyclic elementary components.

Although these matrices are not orthogonal to each other we could determine the strength of the independent components for a given payoff matrix. This calculation is simplified by the fact that the elementary games are described by sparse matrices.

In game theory the separation of the cyclic and hierarchical components can help us extract general relationships between the different types of interactions and their consequences in the macroscopic behavior. It is already known [7] that the hierarchical components are responsible for the possible emergence of social dilemmas in the $n$-strategy potential games whereas the presence of cyclic components prevents the existence of potential as well as the related thermodynamical behavior for the application of logit rules. Now, the voluntary prisoner's dilemma game is selected to demonstrate the applicability of the present approach in the anatomy of games. We emphasize, however, that many concepts and aspects of this decomposition can be adapted for the exploration of other systems characterized by directed graphs.

\section{ACKNOWLEDGMENT}

This work was supported by the Hungarian National Research Fund (OTKA Grant No. TK-120785).
[1] J. von Neumann and O. Morgenstern, Theory of Games and Economic Behaviour (Princeton University Press, Princeton, NJ, 1944).

[2] J. Maynard Smith, Evolution and the Theory of Games (Cambridge University Press, Cambridge, UK, 1982).

[3] M. A. Nowak, Evolutionary Dynamics (Harvard University Press, Cambridge, MA, 2006).

[4] G. Szabó and G. Fáth, Phys. Rep. 446, 97 (2007).
[5] C. P. Roca, J. A. Cuesta, and A. Sánchez, Phys. Life Rev. 6, 208 (2009).

[6] B. Allen and M. A. Nowak, EMS Surv. Math. Sci. 1, 113 (2014).

[7] G. Szabó and I. Borsos, Phys. Rep. 624, 1 (2016).

[8] O. Candogan, I. Menache, A. Ozdaglar, and P. A. Parrilo, Math. Oper. Res. 36, 474 (2011).

[9] G. Szabó, K. S. Bodó, B. Allen, and M. A. Nowak, Phys. Rev. E 90, 042811 (2014). 
[10] G. Szabó, K. S. Bodó, B. Allen, and M. A. Nowak, Phys. Rev. E 92, 022820 (2015).

[11] K. Sigmund, The Calculus of Selfishness (Princeton University Press, Princeton, NJ, 2010).

[12] B. Király and G. Szabó, Phys. Rev. E 95, 012303 (2017).

[13] L. E. Blume, Games Econ. Behav. 5, 387 (1993).

[14] L. E. Blume, Games Econ. Behav. 11, 111 (1995).

[15] D. Monderer and L. S. Shapley, Games Econ. Behav. 14, 124 (1996).

[16] D. Monderer and L. S. Shapley, J. Econ. Theory 68, 258 (1996).

[17] G. Szabó and B. Király, Phys. Rev. E 93, 052108 (2016).

[18] Q. D. La, Y. H. Chew, and B.-H. Soong, Potential Game Theory: Applications in Radio Resource Allocation (Springer, New York, 2016).

[19] B. Bollobás, Modern Graph Theory (Springer, New York, 1998).

[20] R. M. Alston and C. Nowell, J. Econ. Behav. Organ. 31, 357 (1996).

[21] C. Hauert, S. De Monte, J. Hofbauer, and K. Sigmund, Science 296, 1129 (2002).

[22] G. Szabó and C. Hauert, Phys. Rev. E 66, 062903 (2002).

[23] G. Hardin, Science 162, 1243 (1968).

[24] M. Olson, The Logic of Collective Action (Harvard University Press, Cambridge, MA, 1965).
[25] C. Hauert and G. Szabó, Complexity 8, 31 (2003).

[26] L. Sun, P.-j. Lin, and Y.-s. Chen, Physica A 414, 233 (2014).

[27] Q. Yu, R. Chen, and X. Wen, Entropy 17, 1660 (2015).

[28] G. Szabó and J. Vukov, Phys. Rev. E 69, 036107 (2004).

[29] Z.-X. Wu, X.-J. Xu, Y. Chen, and Y.-H. Wang, Phys. Rev. E 71, 037103 (2005).

[30] Y. Chen, S.-M. Qin, L. Yu, and S. Zhang, Phys. Rev. E 77, 032103 (2008).

[31] R. M. May and W. J. Leonard, SIAM J. Appl. Math. 29, 243 (1975).

[32] K. Tainaka, J. Phys. Soc. Jpn. 57, 2588 (1988).

[33] K. I. Tainaka, Phys. Rev. E 50, 3401 (1994).

[34] J. Hofbauer and K. Sigmund, Evolutionary Games and Population Dynamics (Cambridge University Press, Cambridge, UK, 1998).

[35] E. Frey, Physica A 389, 4265 (2010).

[36] A. Szolnoki, M. Mobilia, L.-L. Jyian, B. Szczesny, A. M. Rucklidge, and M. Perc, J. R. Soc. Interface 11, 20140735 (2014).

[37] K. Tainaka, Phys. Lett. A 176, 303 (1993).

[38] H. Gintis, Individuality and Entanglement (Princeton University Press, Princeton, NJ, 2017). 